\title{
El periurbano en México: identificación y caracterización sociodemográfica y territorial
}

\section{The peri-urban in Mexico: Identification and sociodemographic and territorial characterization}

\author{
Salomón González-Arellano \\ Adriana Helia Larralde-Corona \\ Gustavo Manuel Cruz-Bello \\ Departamento de Ciencias Sociales de la Universidad Autónoma \\ Metropolitana, Unidad Cuajimalpa, México
}

Resumen

La producción científica y la política territorial en México se ha construido básicamente a partir de la dicotomía campo-ciudad. En consecuencia, los fenómenos periurbanos no han sido claramente explicados y, las políticas dirigidas a estos espacios no son explícitas o diferenciadas. En ese sentido, este artículo busca aportar información panorámica del periurbano en México. Primeramente, se propone un método para la identificación del periurbano basado en el índice de entropía de Shannon que mide la heterogeneidad del uso del suelo. En un segundo tiempo, el estudio caracteriza el espacio periurbano a través de atributos demográficos, sociales y territoriales. Finalmente, el estudio identifica cuatro dimensiones sociodemográficas y territoriales del espacio periurbano: 1) polarización socio-ocupacional, 2) la oposición entre suelo natural y suelo para el cultivo, 3) urbanización como entorno construido y dinámica demográfica, y 4) marginación social e informalidad residencial. Estas dimensiones sugieren procesos simultáneos diferenciados en el espacio periurbano.

Palabras clave: Espacio periurbano, sistema urbano nacional, dispersión urbana, urbanización.

\section{Abstract}

Scientific production and territorial politics in Mexico have been built basically from the country-city dichotomy. Consequently, peri-urban phenomena have not been clearly explained and the policies addressed at these spaces are not explicit or differentiated. This article aims to provide panoramic information on the peri-urban in Mexico. First, a method is proposed for the identification of the peri-urban based on the Shannon entropy index that measures the heterogeneity of land use. Second, we characterize the peri-urban space by demographic, social and territorial attributes. Finally, the study identifies four sociodemographic and territorial dimensions of the peri-urban space: 1) socio-occupational polarization, 2) the opposition between natural land and cultivated land, 3) urbanization as a built environment, and demographic dynamics, and 4) social marginalization and informal dwelling. These dimensions suggest differentiated simultaneous processes in the peri-urban space.

Keywords: Peri-urban space, national urban system, urban sprawl, urbanization. 


\section{INTRODUCCIÓN}

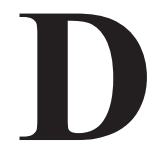

esde mediados del siglo pasado el territorio periurbano acaparó la atención de muchos científicos sociales preocupados por los problemas en las ciudades latinoamericanas. El modelo de desarrollo, inspirado en los principios de la modernidad, significó una enorme presión en el crecimiento de las grandes ciudades, sobre todo hacia las afueras del contorno urbano central, dando lugar al crecimiento de la ciudad en su fase metropolitana. Hoy día son clásicos los estudios críticos sobre la urbanización latinoamericana organizados en torno a la teoría de la dependencia y la marginalidad. De hecho, es en este contexto donde surge la narrativa de la periferia como sinónimo de marginación social. (Lezama, 1998); (Hiernaux y Lindón, 2004).

Medio siglo después de estos primeros análisis, con el ascenso del neoliberalismo y la globalización, se han conformado nuevas formas de exclusión y poblamiento precario en la periferia, los cuales, lejos de cambiar el rumbo heredado del periodo anterior, sólo han contribuido a reforzar el modelo centro-periferia. (Méndez-Lemus y Vieyra, 2014; Aguilar y Lopez, 2016; Cruz-Muñoz y Insunza, 2017).

En el contexto de la ciudad extendida o ciudad-región, el periurbano toma nuevos matices, comparado con el de la ciudad en su fase de crecimiento metropolitano. Recordemos que la teoría del continuum rural-urbano establecía una dicotomía entre las zonas rurales y las zonas urbanas, donde el periurbano funcionaba como una franja de transición que de forma inevitable tendía hacia lo urbano. Con la ciudad-región, se supone que la franja del periurbano es más extensa, y si bien existiría una zona de transición o de incorporación de áreas rurales a la ciudad como resultado del crecimiento natural y social de ésta; también se presentarían espacios híbridos urbano-rurales, que coexistirían dentro de la ciudad sin ninguna tendencia de cambio hacia lo urbano. Situación que necesariamente debería modificar la forma de analizar y de planificar el desarrollo de la ciudad y del campo.

En términos de la política territorial en México, ésta se ha construido a partir de la dicotomía campo-ciudad y, por lo tanto, las políticas dirigidas a los espacios periurbanos no han sido explícitas. Aunque empiezan a ser visibles las iniciativas de intervención planeada en las cuales se concibe a la franja urbano-rural como un territorio singular por el encuentro de dos mundos más o menos diferenciados, con una enorme conflictividad social, pero también con oportunidades para su desarrollo. En este sentido, 
se plantea trabajar en la reducción de la pobreza rural, a través de una vinculación más virtuosa con lo urbano; con la creación de sistemas alimentarios locales, y a través de fomentar la agricultura urbana y periurbana. En cuanto al problema ambiental, se considera que las zonas periurbanas son espacios estratégicos en el proceso de transición socio-ecológica ya que participan de manera significativa en la provisión de servicios ecosistémicos a las ciudades $Y$ se reconoce la trascendencia de concretar mecanismos fuertes de cooperación y coordinación interinstitucional, tanto a nivel sectorial como territorial —urbano y rural—; así como, para el ámbito local, el metropolitano y el regional, con la intención de plantear propuestas pertinentes y políticamente viables.

Es importante comentar que las investigaciones actuales del periurbano han profundizado sobre la identificación y explicación de los innumerables procesos sociales y territoriales que tienen lugar en los distintos periurbanos de las ciudades alrededor del mundo, para ello véase (Avram, 2009; Ravetz, Fertner y Nielsen, 2013). Para el caso de México destacan los trabajos de (Cruz Rodríguez, 2001; Delgado y Galindo, 2009; Vieyra, Méndez-Lemus y Hernández-Guerrero, 2016; Álvaro y Flores, 2016).

A diferencia de los estudios anteriores, para el caso de nuestro país, la presente investigación intenta aportar información a nivel macro y cuantitativa, y así obtener una imagen panorámica del periurbano en México. En este sentido, se plantean dos objetivos:

1. Identificar el espacio periurbano del Sistema Urbano Nacional de México (SUN), el cual estaba conformado para el año 2010 por 384 ciudades $^{1}$. De ahí que una de las aportaciones de la investigación es metodológica.

2. Caracterizar el periurbano, a través de considerar algunos de sus atributos demográficos, sociales y territoriales.

En otras palabras, nos preguntamos: ¿De qué tamaño es el periurbano de las ciudades de México y qué características tiene? Aunque para ello es necesario resolver antes un asunto difícil ¿Cómo definir este tipo de territorios? y, sobre todo, ¿cómo es posible medirlo para todas las ciudades en México?

La metodología para identificar el espacio periurbano del SUN se basa en el índice de entropía de Shannon. Con éste se mide la heterogeneidad

\footnotetext{
1 El SUN para 2010 identifica 384 ciudades de más de 15 mil habitantes, de las cuales 59 son Zonas Metropolitanas, 78 son conurbaciones y 247 son centros urbanos. (Secretaría de Desarrollo Social; Consejo Nacional de Población, 2012).
} 
del territorio en términos del uso del suelo. Las áreas más homogéneas identifican a los territorios en las categorías de urbano o rural. Mientras que las áreas más heterogéneas constituyen el universo dentro del cual se define el periurbano, a través de aplicar un filtro de contigüidad a las aglomeraciones urbanas. Una vez definido el periurbano, se utilizan un grupo de variables relacionadas con la estructura sociodemográfica, económica, de la vivienda y el uso del suelo para describir estos espacios. Finalmente, con el propósito de caracterizar la estructura subyacente del periurbano mexicano se aplica un análisis de componentes principales.

El artículo está estructurado en cinco apartados. Luego de esta introducción, se presenta una revisión teórica sobre el espacio periurbano y su medición. El tercer apartado describe la estrategia metodológica para identificar y caracterizar el periurbano en México. En el cuarto se presentan los resultados; y finalmente, en el quinto, las conclusiones de la investigación.

\section{MARCo TeÓRICO}

El concepto del periurbano o franja rural-urbana es antiguo, Xu Feng (2004) señala que se pueden rastrear definiciones que se remontan a la década de los treinta del siglo pasado. No obstante, continúa el autor, más tarde, a finales de la década de los sesenta, el geógrafo Robin Pryor (1968) elabora una definición muy completa, la cual se retoma para llevar a cabo esta investigación. De acuerdo con Pryor, la franja rural-urbana es una zona de transición del uso del suelo, de las características sociales y, de las demográficas, la cual se localiza entre el área urbana y suburbana construida de la ciudad central y el hinterland rural. Las características sociales y espaciales de esta franja pueden variar en términos zonales y sectoriales, y se modifican a través del tiempo. Dentro de esta zona se encontraría la franja urbana y la franja rural. La primera, es una subzona contigua a la ciudad central, la cual exhibe una densidad ocupacional más alta que la media de la franja rural-urbana total; una proporción alta de suelo residencial, comercial, industrial y vacante; una tasa de crecimiento de la densidad de población más alta, mayor conversión de uso de suelo, y de commuting. La segunda, es la subzona contigua a la franja urbana, con una densidad de ocupación debajo de la media de la franja rural-urbana; una mayor proporción de suelo agrícola; una tasa de crecimiento de la densidad de población más baja, menor de conversión de uso del suelo y de commuting. (Pryor, 1968: 206).

Esta definición incluye dos asuntos importantes del periurbano: i) la mezcla o heterogeneidad que se presenta entre atributos urbanos y rurales; 
y ii) el cambio social de los atributos del tipo rural hacia el urbano. Incluso en la actualidad, con mayor o menor sofisticación, los análisis a nivel macro involucran estas dos ideas. En el presente ejercicio, únicamente se trabaja sobre el primer asunto, sobre la heterogeneidad, y se deja de lado el tema de la transición rural-urbana.

Ahora bien, en relación con la delimitación geográfica de los espacios periurbanos, ha sido un tema que ha captado la atención de diversos investigadores a nivel internacional, Gonçalves et al. (2017) mencionan que se han empleado dos grandes aproximaciones para delimitar de forma espacial al periurbano, la más utilizada es la que emplea la densidad poblacional y la distancia a las zonas urbanas, como se reporta en (OECD, 2009; Piorr, Ravetz y Tosics, 2011; Reginster y Rounsevell, 2006). La otra aproximación emplea variables como tamaño de la población en las zonas construidas, infraestructura y las actividades económicas tal como se describe en Iaquinta y Drescher (2000) y Allen (2003).

Otros estudios han utilizado métodos estadísticos multivariados y univariados, así como variables sociales, económicas y espaciales relacionadas a aspectos eco hidrológicos para definir y categorizar las zonas periurbanas (Danielaini, Maheshwari y Hagare, 2018)

Así, en el contexto del estudio de protección de las casas contra los incendios naturales se han generado diferentes formas de mapear las zonas periurbanas (interfase natural-urbana). En general estas aproximaciones consideran zonas de amortiguamiento alrededor de las casas y la distancia a la que éstas se encuentran de la vegetación natural, que en ese contexto son la fuente de peligro de incendios (Lampin-Maillet et al., 2010).

Bouillon et al. (2014) dividen estas formas de mapear la interfaz urbana-rural dependiendo de la escala espacial: nivel local (comunidades) y nivel global (regiones). La diferencia consiste en variar el buffer alrededor de las viviendas y las distancias a la vegetación natural. Así, a escala local el buffer es más angosto que cuando se hace a escala global. Una modificación consistió en mapear primero los asentamientos y unirlo a una capa de uso del suelo y vegetación para definir el paisaje que las rodea y posteriormente generar los buffers alrededor de los asentamientos que se encuentran a menos de una cierta distancia de la vegetación natural. Un acercamiento similar es definir la interfaz natural-urbana con base en la distribución espacial de las casas y la estructura de la vegetación. Para ellos se clasificaron las zonas urbanas de acuerdo a la densidad de casas y las necesidades de supresión del fuego. Y la estructura de la vegetación se clasificó utilizando un índice de agregación (Herrero-Corral et al., 2012). 
Una vez que se ha delimitado espacialmente el territorio periurbano, es posible hacer una tipificación de las diferentes zonas que lo conforman. Para realizar esta tipificación Gonçalves et al. (2017) emplearon análisis multivariados (componentes principales y análisis de conglomerados) considerando características físicas, económicas y sociales, entre otras movilidad, identidad y estilos de vida, elementos naturales, cobertura del suelo, actividades económicas y funciones espaciales.

A decir verdad, uno de los asuntos más problemáticos para la definición operativa y la caracterización del periurbano, sigue siendo la definición teórica de lo rural y lo urbano. No obstante, en este caso no entramos en la discusión, más bien se retoman los atributos socio-espaciales, al estilo de Pryor (1968), en relación con la mezcla o heterogeneidad que se presenta entre atributos urbanos y rurales clásicos.

Así pues, para los propósitos de este estudio definimos a las zonas periurbanas como aquellos espacios contiguos a las aglomeraciones urbanas y que sirven de interface entre lo urbano y lo rural, es decir, son espacios donde se encuentra y se mezcla la ciudad y el campo. En relación con los atributos que definen lo urbano y lo rural, se utiliza como único criterio de clasificación, el uso del suelo. Es importante mencionar que para clasificar el espacio urbano y rural también se consideraron los atributos: sector de actividad y tipo de vialidad; no obstante, el que mostró mejor comportamiento de acuerdo a nuestros propósitos fue el de uso del suelo. Por lo tanto, un espacio urbano es predominantemente homogéneo en tanto que impera un entorno construido y muy baja presencia de suelo de cultivo y suelo natural. Mientras que un espacio rural es predominantemente homogéneo no construido y dominan los usos con cultivos y áreas naturales. Mientras que uno periurbano es aquel que presenta una mezcla de usos de suelo - urbano y rural-, es decir, tanto construido como con cultivos y zonas con vegetación natural.

\section{Metodología}

La estratégica metodológica se basó en el uso del índice de entropía de Shannon para identificar los espacios periurbanos a partir del grado de diversidad de los usos de suelo. Cabe decir que este índice fue propuesto inicialmente por el autor para cuantificar la entropía. Tomado de las ciencias de la información este índice ha sido usado en diversos campos del conocimiento, entre ellos el de la ecología, donde se emplea ampliamente para medir la diversidad biológica (Hill, 1973; Jost, 2006) en las ciencias geoespaciales donde se ha usado para determinar el grado de diversidad de 
los usos del suelo como un indicador de posible crecimiento de la mancha urbana y los posibles patrones de crecimiento (Ramachandra, Bharath y Sowmyashree, 2015); (Gar-On and Li, 2010).

El estudio abarca el total de las ciudades en México (384 ciudades según el SUN), definidas de acuerdo al Censo de Población y Vivienda 2010. La unidad de análisis es el Área Geoestadística Básica (AGEB) rural y urbana. El procedimiento que se siguió para identificar y caracterizar el periurbano se ordenó en cinco pasos que se ilustran de forma sintética en el diagrama de la Figura 1.

El primer paso se refiere a la recolección y preparación de la información a nivel de las AGEB. Con el propósito de captar la diversidad de diferentes dimensiones del territorio nacional se interrogaron datos tanto del uso de suelo, como de las características sociodemográficas de la población urbana y rural, actividades económicas, infraestructura vial y la delimitación de las zonas urbanas del SUN. ${ }^{2}$ La integración geográfica de estos datos fue a partir de la incorporación de la información a las AGEB urbanas y rurales de todo el territorio nacional. Para el caso del uso del suelo, las categorías se agregaron en tres grandes clases de usos de suelo: construido, cultivo y natural, y se calcularon para cada AGEB la superficie, y el porcentaje de cada clase de uso de suelo; además del número de polígonos para cada clase. De manera similar se agregaron los datos de unidades económicas y los datos de la infraestructura vial.

A partir del tratamiento de estos datos, en el segundo paso, se calculó el índice de entropía tanto para los usos de suelo, como para las actividades económicas y para la infraestructura vial. Para el uso de suelo se realizaron dos tipos de índices de entropía, uno con dos clases: a) construido y b) no construido (cultivado y natural) y; otro con tres clases: i) construido, ii) cultivado y iii) natural. Para las actividades económicas se realizó un índice con tres clases: a) sector primario (agrícola, extractiva, energía), b) sector secundario (sector industrial) y, c) sector terciario (comercio y servicios). Finalmente, para la infraestructura vial: a) caminos y veredas de terracería, b) caminos pavimentados y c) carreteras y autopistas.

\footnotetext{
2 Las fuentes de datos para este proceso son las siguientes: Cartografía geoestadística 2015 versión 6.2. (Instituto Nacional de Estadística y Geografía, 2015a, 2019). Cartas de uso de suelo y vegetación escala 1:50,000 del Sistema Nacional de Información Forestal (Comisión Nacional Forestal, 2015), Directorio Estadístico Nacional de Unidades Económicas (Instituto Nacional de Estadística y Geografía, 2011), Capa vectorial del Sistema Urbano Nacional (Secretaría de Desarrollo Social; Consejo Nacional de Población, 2012), que integra los polígonos de las 384 ciudades a nivel nacional, Infraestructura vial, a partir de tres fuentes a escala nacional: Conjunto de Datos Vectoriales de Carreteras y Vialidades Urbanas, Edición 1.0 (Instituto Nacional de Estadística y Geografía, 2015b); Red de Caminos Rurales del Instituto Mexicano del Transporte (Instituto Mexicano del Transporte, 2016), Red Nacional de Caminos (Instituto Nacional de Estadística y Geografía, 2015c).
} 


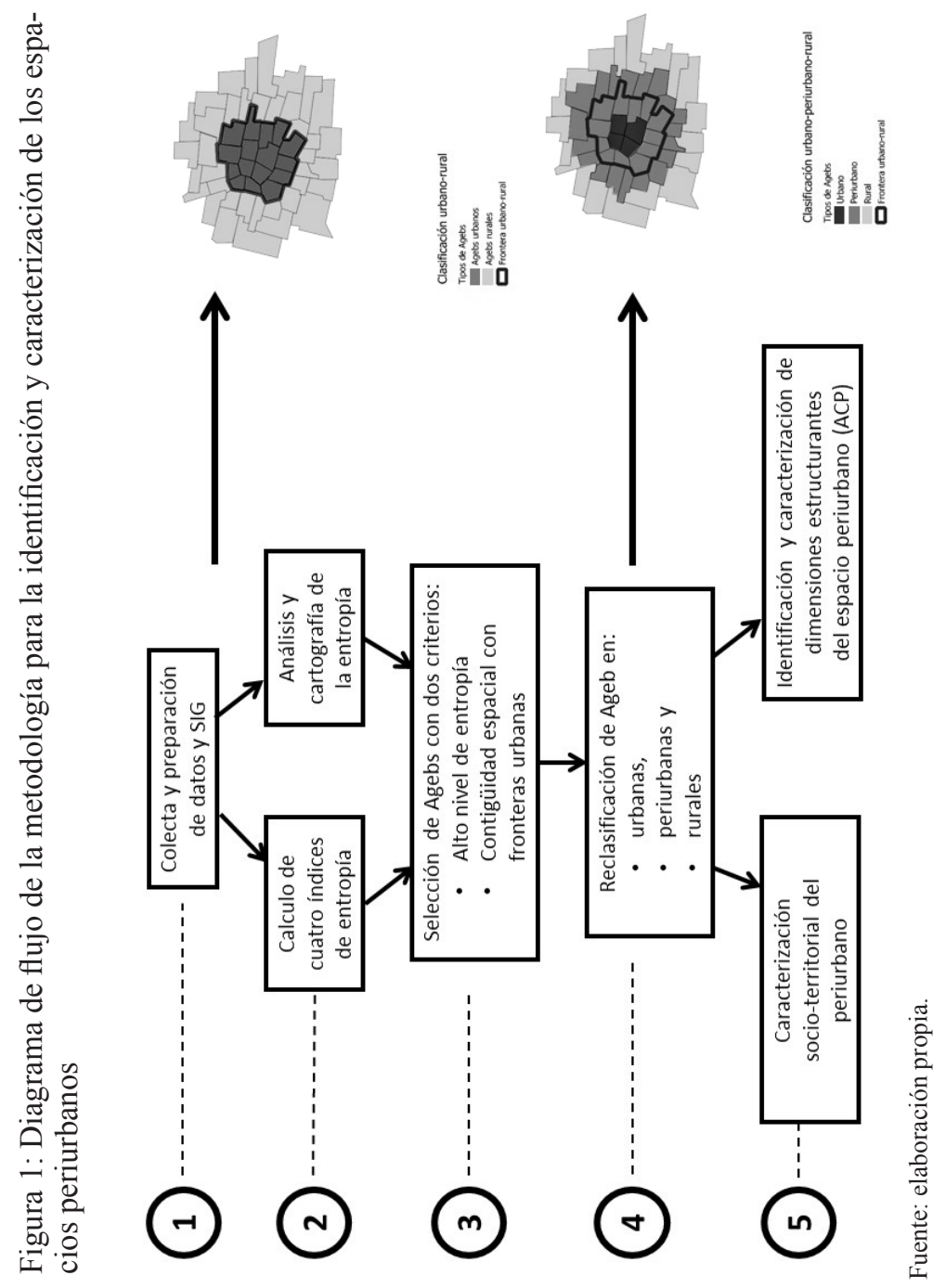


El tercer paso, comprende la selección de las AGEB que corresponden con la acepción conceptual que hemos adoptado en este estudio, es decir, las AGEB que cumplen dos criterios: alto nivel de entropía (con una desviación estándar y más a la media) y que presentan una contigüidad espacial (tanto interna como externa) con las fronteras urbanas. Este segundo criterio permite eliminar numerosas AGEB que presentan alta entropía que se localizan a lo largo de los principales ejes carreteros del país. Esta selección permite realizar el cuarto paso que consiste en la reclasificación de las AGEB y pasar de dos tipos: urbanas y rurales, a tres tipos mutuamente excluyentes: urbanas, rurales y periurbanas.

Por último, el quinto paso se compone del análisis dedicado a la caracterización del espacio periurbano. Además de un análisis descriptivo de un grupo de variables: demográficas, de vivienda y sociales, se realizó un análisis de componentes principales (ACP) con el objeto de identificar las dimensiones subyacentes que componen el espacio periurbano.

\section{Resultados}

Los resultados se ordenan en tres apartados. El primero, contiene el análisis de la entropía y la identificación del periurbano. Cabe mencionar que, aunque se trata de los resultados, éstos tienen un carácter metodológico, al menos en lo que toca al análisis de entropía. En el segundo, se presenta la caracterización socioterritorial del espacio periurbano nacional. Finalmente, el tercero contiene el Análisis de Componentes Principales (ACP) y la interpretación de las dimensiones o componentes.

\section{Análisis de entropía e identificación del periurbano}

El país abarca una superficie de 1.96 millones de kilómetros cuadrados. El marco geoestadístico nacional subdivide el territorio en 77,789 AGEB. De este total, 60,324 son AGEB urbanas (77.5 por ciento) y 17,465 rurales (22.5 por ciento), lo cual corresponde a la clasificación de la población rural o urbana según tamaño de la localidad del INEGI. ${ }^{3}$ No obstante, si pensamos en términos de la superficie, los datos indican una situación contraria, dado que las AGEB rural abarcan 98.7 por ciento del territorio de nuestro país (Instituto Nacional de Estadística y Geografía, 2019).

Ahora bien, en relación con el análisis de entropía, se calcularon dos índices para el uso del suelo, para dos y para tres clases de uso del suelo, respectivamente. El índice que presenta los valores con mayor variabilidad

\footnotetext{
${ }^{3}$ De acuerdo con el INEGI, una población se considera rural cuando tiene menos de 2,500 habitantes, mientras que la urbana es aquella donde viven 2,500 y más personas.
} 
y que ofrece una mejor discriminación del territorio, tanto para el nivel nacional como para las AGEB urbanas y rurales, son aquellos estimados a partir de tres clases de uso de suelo. Estos resultados sugieren que el índice de entropía es un predictor adecuado para medir la heterogeneidad del territorio. Además de este comportamiento estadístico, el patrón geográfico de los indicadores observados por medio de un análisis cartográfico refleja una tendencia de mayor entropía en las zonas periféricas de las ciudades y a lo largo de los principales ejes carreteros del país. Los mapas de las figuras 2 y 3 muestran el índice de entropía para varias ciudades y zonas metropolitanas del país. En éstos se observa que, al interior de las zonas urbanas el índice de entropía es generalmente bajo, es decir, con un uso de suelo muy homogéneo, que para el caso de lo urbano se trata del espacio construido. Además de las zonas urbanas, los espacios lejanos a las ciudades, o las zonas naturales protegidas cercanas a las ciudades, presentan igualmente valores bajos en el índice de entropía, esta vez por ser espacios básicamente con uso de suelo natural o de cultivo, como es, por ejemplo, la zona que abarca el Parque Nacional Iztaccíhuatl-Popocatépetl, al suroriente de la ZM Valle de México (ver Figura 2).

Los valores con alta entropía (colores oscuros en los mapas) se localizan en términos generales en las periferias de las zonas urbanas y en ejes carreteros. El caso del corredor industrial del Bajío; que va de la ZM de Querétaro a la ZM de León es un buen ejemplo de ejes carreteros con alta diversidad de usos de suelo (ver Figura 3, último recuadro).

Con el fin de acotar el periurbano, se seleccionaron todas las AGEB urbanas y rurales que estuvieran en contigüidad con la frontera de cada una de las ciudades del Sistema Urbano Nacional y, se eliminaron aquellas que se localizaron sobre los ejes carreteros, aunque también presentaban altos índices de entropía. El mapa de la Figura 4 ilustra para la región centro del país la recategorización de las AGEB urbanas, periurbanas y rurales.

\section{Descripción del espacio periurbano}

Los resultados indican que el espacio periurbano abarca 16 por ciento de las AGEB del país $(12,681)$. Cabe mencionar que en términos de la proporción de población ocurre una situación similar, pues 13.5 por ciento de la población en México tendría residencia periurbana, alrededor de 15 millones de habitantes. (Tabla 1). 
El periurbano en México: Identificación y caracterización ... / S. GONZÁLEZ ARELLANO et al.

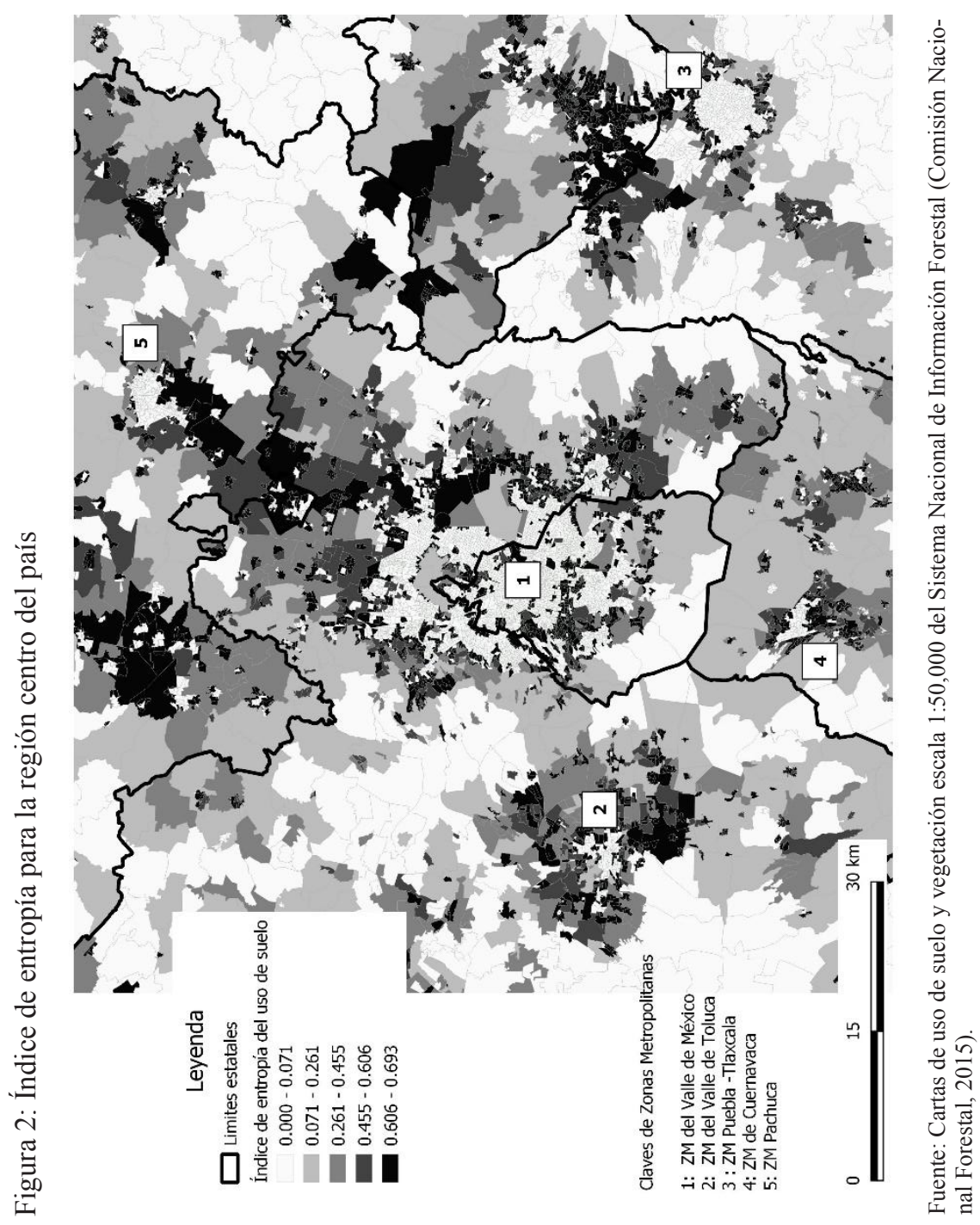




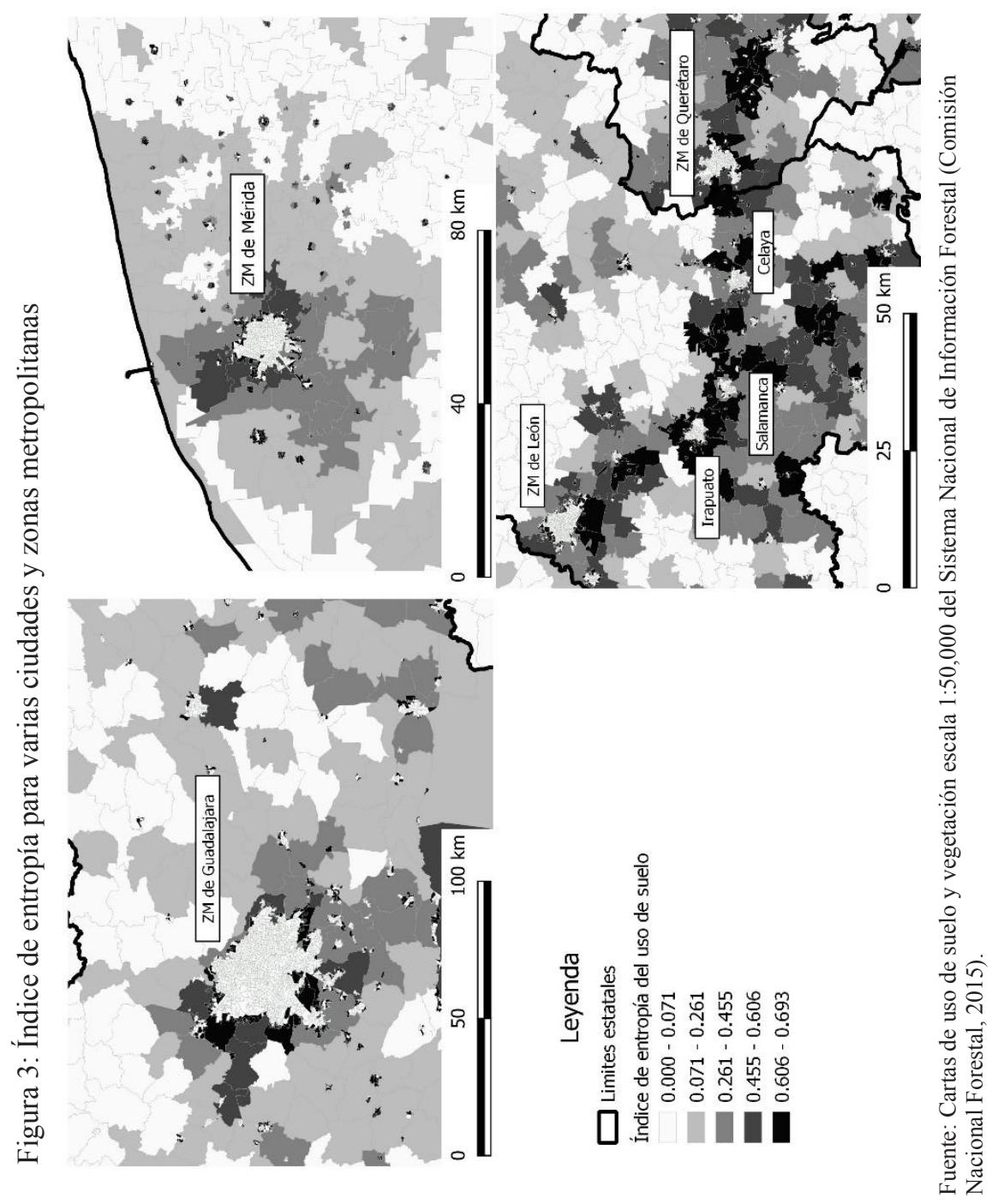


El periurbano en México: Identificación y caracterización ... / S. GONZÁLEZ ARELLANO et al.

Figura 4: Recategorización de zonas urbanas, periurbanas y rurales

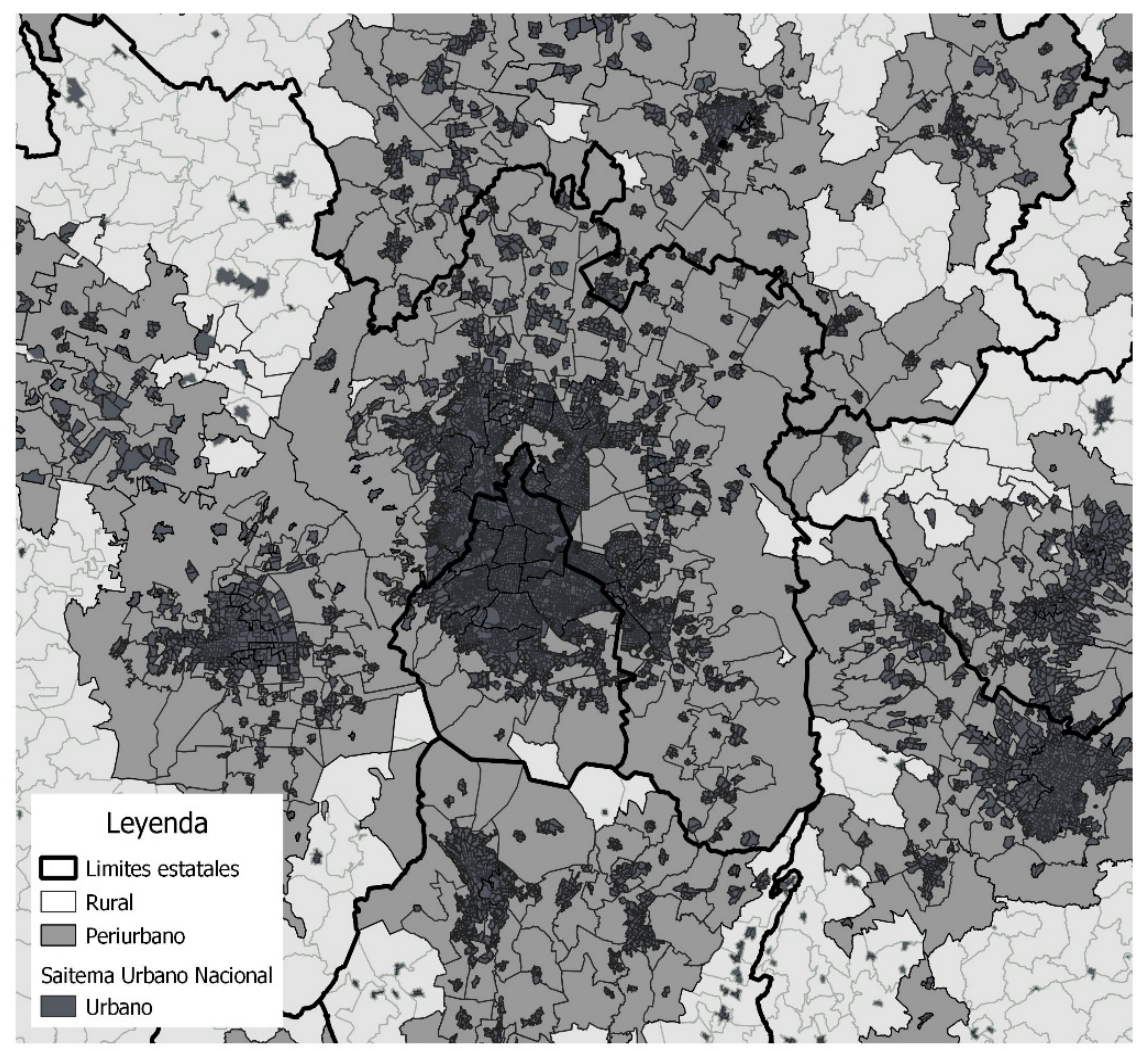

Fuente: elaboración propia a partir de resultados del análisis.

Tabla 1: AGEB totales, urbanas, periurbanas y rurales: población, superficie y densidad

\begin{tabular}{crrrr}
\hline Características & Total & Urbano & Periurbano & Rural \\
\hline Número de AGEB & 77,789 & 47,643 & 12,681 & 17,465 \\
$\%$ & 100.0 & 61.2 & 16.3 & 22.4 \\
Población & $112^{\prime} 251,268$ & $71^{\prime}, 550,573$ & $15^{\prime} 164,253$ & $25^{\prime} 536,442$ \\
$\%$ & 100.0 & 63.7 & 13.5 & 22.7 \\
Superficie (ha) & $195^{\prime} 623,988.8$ & $1^{\prime}, 711,799.4$ & $616,414.3$ & $1933^{\prime} 295,775.1$ \\
$\%$ & 100.0 & 0.9 & 0.3 & 98.8 \\
Densidad (ha) & 37.7 & 53.2 & 48.61 & 0.24 \\
\hline
\end{tabular}

Fuente: elaboración propia. 
En cuanto a la extensión del periurbano, a juzgar por el tamaño de nuestro país, se podría decir que es mínimo, 0.3 por ciento. Lo mismo sucede con la urbana, con poco menos de uno por ciento de la superficie del territorio nacional (0.9 por ciento) No obstante, si comparamos el periurbano con la superficie urbana, resulta significativo el hecho de que 26.5 por ciento corresponde al periurbano y, 73.5 por ciento al espacio urbano. Conviene subrayar que se trata de un promedio y la situación del balance urbano-periurbano varía de forma significativa para cada una de las áreas urbanas en México.

Tabla 2: Características sociales del espacio nacional, urbano, periurbano y rural

\begin{tabular}{|c|c|c|c|c|c|}
\hline Núm. & Variables & Nacional & Urbano & Periurbano & Rural \\
\hline 1 & $\begin{array}{l}\text { Proporción dependencia } \\
\text { demográfica }\end{array}$ & 43.37 & 40.10 & 40.26 & 54.88 \\
\hline 2 & Promedio de hijos vivos & 2.12 & 2.07 & 1.96 & 2.37 \\
\hline 3 & $\begin{array}{l}\text { Total de viviendas } \\
\text { particulares }\end{array}$ & $34^{\prime} 988,335$ & $22^{\prime} 483,385$ & $4^{\prime} 829,738$ & $7^{\prime} 675,212$ \\
\hline 4 & $\begin{array}{l}\text { Promedio de ocupantes } \\
\text { por vivienda }\end{array}$ & 3.35 & 3.48 & 3.38 & 2.99 \\
\hline 5 & $\begin{array}{l}\text { Proporción de vivienda } \\
\text { con } 3 \text { y más cuartos }\end{array}$ & 70.10 & 72.87 & 67.93 & 63.23 \\
\hline 6 & $\begin{array}{l}\text { Proporción de vivienda } \\
\text { con excusado }\end{array}$ & 92.18 & 95.02 & 94.25 & 81.75 \\
\hline 7 & $\begin{array}{l}\text { Proporción de población } \\
\text { de } 18 \text { a } 24 \text { años que asiste } \\
\text { a la escuela }\end{array}$ & 24.16 & 28.39 & 23.02 & 12.31 \\
\hline 8 & $\begin{array}{l}\text { Grado promedio } \\
\text { de escolaridad }\end{array}$ & 6.79 & 7.74 & 6.97 & 4.07 \\
\hline 9 & $\begin{array}{l}\text { Proporción de población } \\
\text { sin derechohabiencia }\end{array}$ & 31.51 & 31.86 & 32.75 & 29.62 \\
\hline 10 & $\begin{array}{l}\text { Proporción de población } \\
\text { de } 3 \text { y más que habla } \\
\text { lengua indígena }\end{array}$ & 5.64 & 4.53 & 4.02 & 9.94 \\
\hline
\end{tabular}

Fuente: elaboración propia.

Por consiguiente, pensaríamos que es en este 26 por ciento del territorio $(616,414 \mathrm{ha})$, donde se presenta la mayor complejidad, derivada del enfrentamiento entre lo urbano y lo rural; el espacio del cambio social acelerado, sobre todo, de las actividades agropecuarias y silvícolas, hacia el sector secundario y terciario. Asimismo, es el territorio donde prolifera la ocupación residencial de zonas de cultivo; o bien, con valor ambiental 
(catalogadas o no); así como, de alto riesgo. Pero también es el espacio que alude a la ciudad-región, la ciudad difusa, el poblamiento suburbano, etc. donde prevalece una forma de poblamiento más disperso que el urbano central y es más diverso.

Ahora, en lo que se refiere a las características socio-demográficas y de la vivienda del espacio periurbano, se muestran algunos datos censales importantes. La dependencia demográfica, es decir, la parte de la población total que es menor de 15 años o mayor de 65 años, como un indicador del soporte social necesario para la población inactiva, es de 40.3 en la franja periurbana; similar a la urbana, 40.1. Mientras que para el territorio rural es claramente mayor, de 54.9. En cuanto a la fecundidad, la media de las AGEB periurbanas es de dos hijos, ligeramente inferior a la urbana (2.1) y a la rural, que es de 2.1 (Tabla 2).

En lo que corresponde a las características de las viviendas, se observa que el periurbano cuenta con un total de 4,829,738 viviendas ocupadas, lo cual representa casi 14 por ciento del total nacional. Es aquí donde podemos imaginar una gran diversidad de formas de producir y habitar el territorio, que gravitan en torno a las características urbanas y las rurales.

En términos de número de ocupantes por vivienda, las zonas periurbanas muestran en promedio 3.4 personas; en tanto que las urbanas reportan una media de 3.5. El porcentaje de viviendas con tres cuartos o más, es de 67.9 por ciento para las AGEB periurbanas, mientras que la media para el espacio urbano es de 72.9 por ciento. En cuanto a la proporción de viviendas con excusado, como una medida de bienestar para la población, se aprecia que 94.2 por ciento cuentan con el servicio, similar al porcentaje en el medio urbano, 95 por ciento; mientras que las zonas rurales muestran mayor rezago, pues su porcentaje es de 81.7.

Conviene detenernos aquí para comentar que, si bien es cierto que el periurbano presenta un nivel de mezcla (rural-urbano) alto, según el índice de Shannon, las características de la vivienda son muy similares. Esta situación resulta a primera vista paradójica e invita a profundizar sobre la dimensión residencial; ya sea en términos de los indicadores que se utilizan, la escala y la unidad de análisis apropiadas, etcétera.

Ahora bien, en relación con la educación, el periurbano reporta un ligero atraso con respecto a las zonas urbanas. La población con edad de estudios superiores y que asiste a la escuela representa una media de 23 por ciento; mientras que para las urbanas es de 28 por ciento. En tanto que en los espacios rurales desciende hasta 12 por ciento. En el mismo sentido, el grado promedio de escolaridad, los espacios periurbanos indican que éste 
es de siete años, mientras que en las AGEB urbanas es de 7.8. Por su parte, los espacios rurales muestran un marcado rezago, con 4.1 años.

En cuanto a la población sin servicios de salud, como uno de los indicadores importantes para medir las condiciones de vida, sorprende el hecho de que es similar para los tres tipos de territorios, muy cerca de 30 por ciento. Claramente la salud, como dimensión de análisis, es otro tema que requiere de mayor estudio, porque resulta contraintuitivo; sobre todo si pensamos en la calidad de vida en el espacio rural.

Finalmente, en cuanto a la población que habla una lengua indígena, la proporción en el periurbano es de cuatro por ciento, semejante al urbano (4.5 por ciento); mientras que en el espacio rural es del doble (nueve por ciento).

En síntesis, se puede decir que el periurbano presenta un rezago social comparado con el espacio urbano; no obstante, éste es mínimo y acotado a ciertos indicadores, como la educación. La diferencia se observa, claramente, entre el espacio urbano y periurbano, por un lado; y el rural, por el otro.

\section{Caracterización síntesis del espacio periurbano}

Con el objeto de construir una síntesis de las características sociales, residenciales y físicas del espacio periurbano nacional se desarrolló un análisis de componentes principales (ACP) para las AGEB periurbanas. Este nos permite identificar las principales dimensiones que discriminan este espacio y caracterizarlo. Para este efecto, se seleccionaron las variables que mejor representan diferentes aspectos de la población, la vivienda y el medio físico.

Del análisis de componentes principales cuatro factores dan cuenta de 64.8 por ciento de la varianza total. Los factores comparten una distribución bastante equilibrada entre ellos: el primer factor aporta 18.9 por ciento, el segundo 17.2 por ciento, el tercer factor representa 14.7 por ciento, $\mathrm{y}$ el cuarto, con 14.0 por ciento de la varianza total (Tabla 3).

Una revisión de las notas en factor para cada variable utilizada en el análisis permite iniciar una interpretación de la conformación de los cuatro componentes o factores. El primer factor se compone principalmente por la contribución de tres variables de ámbitos distintos; la proporción de población dependiente con un valor de -0.902, proporción de población ocupada con 0.789 y proporción de población nacida en la entidad con 0.695. Este factor presenta una oposición de las variables, población dependiente con respecto a la población ocupada y la población nacida en la entidad. 
El periurbano en México: Identificación y caracterización ... / S. GONZÁLEZ ARELLANO et al.

Tabla 3: Composición de los factores del ACP

\begin{tabular}{|c|c|c|c|c|c|c|}
\hline \multicolumn{7}{|c|}{ Varianza total explicada } \\
\hline \multirow[t]{2}{*}{ Componente } & \multicolumn{3}{|c|}{$\begin{array}{c}\text { Sumas de las saturaciones } \\
\text { de la extracción }\end{array}$} & \multicolumn{3}{|c|}{$\begin{array}{c}\text { Suma de las saturaciones } \\
\text { de la rotación }\end{array}$} \\
\hline & Total & $\begin{array}{r}\% \text { de la } \\
\text { varianza }\end{array}$ & $\begin{array}{r}\% \\
\text { acumulado }\end{array}$ & Total & $\begin{array}{r}\% \text { de la } \\
\text { varianza }\end{array}$ & $\begin{array}{r}\% \\
\text { acumulado }\end{array}$ \\
\hline 1 & 2.73 & 22.747 & 22.747 & 2.271 & 18.923 & 18.923 \\
\hline 2 & 2.218 & 18.48 & 41.228 & 2.065 & 17.21 & 36.132 \\
\hline 3 & 1.684 & 14.031 & 55.258 & 1.761 & 14.677 & 50.809 \\
\hline 4 & 1.144 & 9.537 & 64.795 & 1.678 & 13.986 & 64.795 \\
\hline
\end{tabular}

Fuente: elaboración propia.

Esto nos sugiere que se trata de una dimensión socioeconómica que discrimina el espacio periurbano entre aquellos que presentan una inserción laboral (con signo positivo) contra aquellos espacios que existe una fuerte carga de dependencia demográfica y población desocupada. Este factor puede ser denominado como una dimensión que refleja la polarización socio-ocupacional de los espacios periurbanos (ver Tabla 4).

Tabla 4: Valores de las notas en factor del ACP

\begin{tabular}{lcccc}
\hline & \multicolumn{5}{c}{ Componente } \\
Variable & 1 & 2 & 3 & 4 \\
\hline Prop. Población dependiente & $-0-902$ & $-0-136$ & $-0,118$ & -0.009 \\
Promedio de hijos vivos & -0.073 & -0.104 & -0.774 & 0.070 \\
Grado promedio de escolaridad & 0.082 & 0.068 & 0.746 & -0.393 \\
Prop. de población nacida en la entidad & 0.695 & 0.177 & -0.371 & 0.286 \\
Prop. de población en hogares indígenas & -0.079 & -0.163 & 0.072 & 0.682 \\
Prop. de población ocupada & 0.789 & -0.053 & 0.250 & -0.088 \\
Promedio de ocupantes por vivienda & 0.214 & 0.258 & -0.132 & 0.592 \\
Prop. de viviendas con servicios & 0.365 & 0.110 & 0.143 & -0.540 \\
Prop. de población sin derechohabiencia & 0.379 & -0.031 & -0.001 & 0.541 \\
Porcentaje suelo natural & -0.076 & -0.968 & -0.147 & 0.033 \\
Porcentaje suelo construido & 0.032 & 0.001 & 0.571 & 0.151 \\
Porcentaje suelo cultivo & 0.069 & 0.977 & 0.018 & -0.068 \\
\hline
\end{tabular}

Fuente: elaboración propia. 
El factor 2 da cuenta de 17.21 por ciento de la varianza total y se compone principalmente por la contribución de dos variables fuertemente opuestas; con signo negativo está la variable porcentaje de suelo natural con una nota de -0.968 y con signo positivo la variable porcentaje de uso de suelo de cultivo con 0.977. La fuerte covarianza con signos opuestos de estas dos variables sugiere una especie de competencia por el uso del suelo entre el suelo natural y el de cultivo. El resto de las variables contribuyen con notas en factor de manera débil por lo que permite elaborar una claridad en su interpretación como la oposición suelo cultivo vs. suelo natural.

Con una contribución de 14.67 por ciento a la varianza total, el tercer factor se compone por la participación principalmente de dos variables que covarían en sentido opuesto: con signo negativo está la variable promedio de hijos nacidos con -0.774, y con signo positivo, el grado promedio de escolaridad con 0.746. La contribución de estas dos variables sugiere una asociación conocida entre baja fecundidad y niveles de escolaridad de la población. Aunado a esas dos variables, con menor fuerza covaría de manera positiva la variable porcentaje de usos de suelo construido con 0.571 . En este sentido, una interpretación de las variables que contribuyen en la construcción de este factor; baja fecundidad con escolaridad y presencia de construcciones sugiere que se trata de espacios en proceso de ser urbanizados.

El cuarto factor aporta 13.98 por ciento de la varianza total, y refleja por su parte, la presencia de hogares indígenas (proporción de hogares indígenas +0.682 ) y viviendas precarias; con signo negativo viviendas con servicios (-0.540) y con signo positivo número de ocupantes por vivienda (0.592). Además de la covarianza de estos dos tipos de variables, también participa con signo positivo la variable población sin derechohabiencia (0.541). Hay que tomar en cuenta que del total de los hogares que residen en las AGEB periurbanas 4.02 por ciento son hogares indígenas, el porcentaje de vivienda con servicios es en promedio de 55.06 y que el porcentaje de población sin derechohabiencia a servicios de salud es en promedio de 33.77. Este factor puede ser interpretado como las condiciones de marginación e informalidad socioresidenciales del espacio periurbano.

\section{Conclusiones}

La dinámica territorial en México se ha caracterizado por dos procesos importantes: i) una gran concentración de la población en un grupo reducido de grandes ciudades; y ii) una importante dispersión con cientos de miles de localidades rurales. En relación con la ciudad y la forma urbana, 
se observa un proceso de dispersión y fragmentación, sobre todo en los espacios periurbanos de buena parte de las ciudades, no sólo en las grandes urbes como la Ciudad de México, Guadalajara o Monterrey (Secretaría de Desarrollo Social, 2011; González Arellano y Larralde, 2019).

El propósito de la investigación fue proporcionar una base empírica a nivel macro, es decir, para todo el sistema urbano de México, conformado para el año 2010 por 384 ciudades, de las características sociales y territoriales del periurbano. La primera tarea fue esencialmente metodológica, plantear una definición que fuera operativa para medir todo el país. Para ello se empleó la noción de periurbano clásica, la cual retoma la dicotomía campo y ciudad, donde el periurbano se caracteriza por la mezcla de los dos tipos de hábitat. De ahí que, la intención metodológica fuera medir la heterogeneidad. Para ello se utilizó el índice de entropía de Shannon aplicado al uso del suelo. Los análisis de entropía realizados demostraron que, es el entorno periférico de las ciudades y en los grandes ejes carreteros del país donde coexisten diferentes usos de suelo, tanto urbano como rural. Finalmente, se adoptó un criterio de contigüidad espacial con las fronteras urbanas para llegar a la definición del periurbano.

Así pues, a la pregunta que nos planteamos al inicio de la investigación: ¿de qué tamaño es el territorio periurbano? Se encontró que, en términos de su población, representa 13.5 por ciento del total, alrededor de $15 \mathrm{mi}$ llones de habitantes; y tiene una extensión de 616,414 hectáreas, lo que significa, menos 0.3 por ciento de la superficie total de nuestro país. No obstante, si nos enfocamos exclusivamente en la ciudad y el periurbano, se observa que, el área urbana representa 74 por ciento; y la periurbana, 26 por ciento. En términos de población significa: 82 por ciento para la primera y 17.5 por ciento en el periurbano.

A reserva de profundizar en estos resultados, el tamaño del periurbano da lugar a suponer que, de acuerdo con la teoría del Continuum rural-urbano, una parte de éste transitará hacia lo urbano; no obstante, la otra, y es lo que nos interesa subrayar, es un espacio híbrido urbano-rural que forma parte de la ciudad-región del sistema urbano nacional actual.

Esto nos da una idea de la magnitud de los conflictos socioterritoriales actuales en México, asociados al cambio del uso del suelo en el entorno regional de la ciudad, donde actores citadinos se imponen a los intereses y actores del campo. Asimismo, a las contradicciones relacionadas con la política territorial actual, donde predomina una visión dicotómica cerrada —urbano vs rural—y que choca la idea de los espacios híbridos y de flujos. 
En otro orden de ideas, los resultados sobre el tamaño del periurbano, cuestionan algunas de las afirmaciones que hicimos al inicio de la investigación. En concreto, se dijo que el periurbano jugaba un papel importante para enfrentar el problema ambiental de la ciudad. Ahora que sabemos que el espacio periurbano abarca 0.3 por ciento del territorio nacional; y que, incluso el urbano no llega ni a uno por ciento, parece más sensato que su importancia debiera acotarse a la escala geográfica del espacio local urbano y de la "región urbana", y para ciertos problemas ambientales. Porque si se trata de enfrentar el problema ambiental de la ciudad en su justa dimensión, sería necesario recurrir a otra escala, y a nociones más adecuadas, como sería la huella ecológica, o sus versiones más acotadas, como la huella de carbono o la hídrica. Dicho sea de paso, lo señalamos como un reclamo a cierto urbanismo que sataniza el poblamiento urbano periférico como el mal de las ciudades, por la transformación/destrucción del suelo rural y natural, cuando éste es mínimo, comparado con la huella ecológica de cada uno de los citadinos.

Ahora bien, en lo que corresponde a las características sociales del periurbano, los datos censales muestran una población que se parece a la urbana. Y si bien es cierto que presenta un ligero rezago en las condiciones de vida, es poco significativo cuando se compara con la población rural. En este sentido, si bien se corrobora la idea de la periferia urbana como un espacio de mayor marginación social, como se asentó en la introducción, realmente conviene profundizar y refinar el análisis para discriminar mejor las diferencias según dimensiones: demográfica, económica, de la vivienda, de la salud, etcétera; y por sector espacial. Pues es claro que, como ya se ha señalado en otras investigaciones, las condiciones de vida y la calidad del espacio construido, además de la "determinación" centro-periferia, que responde en gran medida a la renta de localización y al proceso de consolidación material de las edificaciones; también tiene un componente sectorial muy importante — norte, sur, oriente, poniente —, que responde a otros factores (paisaje, riqueza ambiental, normas urbanas, vulnerabilidad, etcétera); así como a la historia particular de la urbanización de cada una de las ciudades.

Por otra parte, en relación con el análisis estadístico de componentes principales, se identificaron cuatro dimensiones que estructuran y discriminan el espacio periurbano de México. Estas dimensiones dan cuenta de tres ámbitos independientes entre ellos: i) una dimensión que refleja la polarización socio-ocupacional, ii) la oposición-competencia entre el suelo natural y el suelo para cultivo, iii) la urbanización entendida como el desarrollo 
del medio construido y una dinámica demográfica, y iv) las condiciones de marginación e informalidad socioresidencial. Estas dimensiones sugieren procesos simultáneos diferenciados en el espacio en torno a las ciudades.

Los resultados nos permiten elaborar una imagen más concreta y precisa del espacio periurbano en México. En términos del tamaño, el periurbano mexicano puede ser una categoría espacial importante que merece considerar una mayor atención académica y, sobre todo, de la política pública territorial.

\section{Agradecimientos}

Esta investigación fue financiada por la beca SEMARNAT-2015-01-263102 (Consejo Nacional de Ciencia y Tecnología / Secretaría de Medio Ambiente y Recursos Naturales), para el proyecto titulado "Análisis de la Vulnerabilidad y Resiliencia al Cambio Climático en Sistemas Socio-ecológicos Periurbanos. Queremos agradecer a Miguel Uribe, Laura Quiroz y Socorro Flores quienes participaron en la recolección y análisis de los datos.

\section{REFERENCIAS BIBLIOGRÁFICAS}

Aguilar, A. G. y López, F. M., 2016, "Espacios de pobreza en la periferia urbana y suburbios interiores de la Ciudad de México: Las desventajas Acumuladas.", en EURE (Santiago), enero-2016. 42(125). Fecha de consulta: 19/08/2020. pp. 5-29. Disponible en https://doi.org/10.4067/S0250-71612016000100001.

Allen, A., 2003, "La interfase periurbana como escenario de cambio y acción hacia la sustentabilidad del desarrollo", en Cuadernos del Cendes, 53. Fecha de consulta: 19/08/2020. p. 15. Disponible en http://ve.scielo.org/scielo.php?scrip$\mathrm{t}=$ sci_arttext\&pid=S1012-25082003000200002

Álvaro, J. y Flores, H., 2016, "Capital cultural y estrategias educativas en hogares periurbanos. Un estudio comparativo en tres localidades del centro de México", en Perfiles Educativos. 38(154). Fecha de consulta: 20/08/2020, pp. 443-450. Disponible en http://perfileseducativos.unam.mx/iisue_pe/index.php/perfiles/issue/ view/4454/pdf154

Avram, S., 2009, "The position of rural-urban fringe in the framework of human settlement system", en Forum Geografic. Studdi și cercetäri de geografie și protectia mediului. 8(8). Fecha de consulta 19/08/2020 pp. 139-145. Disponible en http://forumgeografic.ro/2009/250/

Bouillon, C.; Fernandez M; Sirca, C.; Fierro B; Casula, F.; Vila, B; Long, M.; Pellizzaro, G.; Arca, B.; Tedim, F; Trebini, F; Derudas, A; Cane, S., 2014, “A tool for mapping rural-urban interfaces on different scales, in advances in forest fire research. Chapter 3 - Fire Management - A tool for mapping rural-urban interfaces on different scales", in Advances in forest fire research. Imprensa da Universidade de Coimbra: Coimbra, Portugal, pp. 611-625. 
Comisión Nacional Forestal, 2015, Cartas de Uso de Suelo y Vegetación escala 1:50,000. Fecha de revisión: 2020. Fecha de consulta: 19/08/2020. Disponible en https://www.inegi.org.mx/temas/usosuelo/default.html\#Herramientas

Cruz-Muñoz, F. y Insunza, G., 2017, “Construcción del hábitat en la periferia de la Ciudad de México. Estudio de Caso en Zumpango", en EURE (Santiago), 43(129). Fecha de consulta: 19/08/2020. pp. 187-207.

Cruz Rodríguez, M. S., 2001, Propiedad, poblamiento y periferia rural en la Zona Metropolitana de la Ciudad de México. Programa Editorial de la Red de Investigación Urbana, Universidad Autónoma Metropolitana: Ciudad de México, México.

Danielaini, T. T., Maheshwari, B. y Hagare, D., 2018, “Defining rural-urban interfaces for understanding ecohydrological processes in West Java, Indonesia: Part I. Development of methodology to delineate peri-urban areas", en Ecohydrology and Hydrobiology. European Regional Centre for Ecohydrology of the Polish Academy of Sciences. 18(1). Fecha de consulta: 19/08/2020. pp. 22-36. Disponible en 10.1016/j.ecohyd.2017.11.006

Delgado, J. y Galindo, C., 2016, "Los espacios emergentes de la dinámica rural-urbana.", Problemas Del Desarrollo", en Revista Latinoamericana de Economía. 37(147). Fecha de consulta: 19/08/2020 Disponible en http://www.revistas. unam.mx/index.php/pde/article/view/7639/7128.

Gar-On, Y. y Li, X., 2010, "Modelling sustainable urban development by the integration of constrained cellular automata and GIS", en International Journal of Geographical Information Science. 14(2). Fecha de consulta: 19/08/2020. pp. 131-152. Disponible en https://doi.org/10.1080/136588100240886.

Gonçalves, J. et al., 2017, "Differentiating peri-urban areas: A transdisciplinary approach towards a typology", in Land Use Policy. Fecha de consulta: 19/08/2020. pp. 331-341. Disponible en 10.1016/j.landusepol.2017.01.041

González Arellano, S. y Larralde, A. H., 2019, "La forma urbana actual de las zonas metropolitanas en México: indicadores y dimensiones morfológicas", en Estudios Demográficos y Urbanos. 34(1). Fecha de consulta: 19/08/2020. pp. 11-42. Disponible en https://doi.org/10.24201/edu.v34i1.1799

Herrero Corral, G., Jappiot, M., Bouillon, C., y Long-Fournel, M., 2012, “Application of a geographical assessment method for the characterization of wildland-urban interfaces in the context of wildfire prevention: A case study in western Madrid", en Applied Geography. 35(1-2). Fecha de consulta: 19/08/2020. pp: 60-70. Disponible en https://doi.org/10.1016/j.apgeog.2012.05.005

Hiernaux, D. y Lindón, A., 2004, "La periferia: voz y sentido en los estudios urbanos", en Papeles de Población. 10(42). Fecha de consulta: 19/08/2020. pp. 101123. Disponible en https://www.redalyc.org/articulo.oa?id=11204205

Hill, M. O., 1973, "Diversity and Evenness: A Unifying Notation and Its Consequences", en Ecology: Ecological Society of America. 54(2). Fecha de consulta: agosto-2020. pp. 427-432. Disponible en: https://doi.org/10.2307/1934352 


\section{El periurbano en México: Identificación y caracterización ... / S. GONZÁLEZ ARELLANO et al.}

Iaquinta, D. L. y Drescher, A. W., 2000, "Defining the peri-urban: rural-urban linkages and institutional connections", en Land Reform, Land Settlement and Cooperatives. 2(2000). Fecha de consulta: 19/08/2020. pp. 8-26. Disponible en http://portal.geographie.uni-freiburg.de/forschungsprojekte/indigenoveg/Background1PeriurbanTypology.pdf

Instituto Mexicano Del Transporte, 2019, Red de Caminos Rurales. Consulta 10/08/2020. Disponible en: https://www.gob.mx/imt

Instituto Nacional de Estadística y Geografía, 2017, Directorio Estadístico Nacional de Unidades Económicas 2011-2017. Consulta 8/08/2020. Disponible en https://www.inegi.org.mx/rnm/index.php/catalog/335/study-description?idPro=

Instituto Nacional de Estadística y Geografía, 2014, Cartografía Geoestadística versión 6.2. Consulta 8/08/2014. Disponible en https://datos.gob.mx/busca/dataset/cartografia-geoestadistica-urbana-y-rural-amanzanada-planeacion-de-la-encuesta-intercensal-2015

Instituto Nacional de Estadística y Geografía, 2015, Conjunto de datos Vectoriales de Carreteras y Vialidades Urbanas. 8/08/2020. Disponible en https://datos.gob. $\mathrm{mx} / \mathrm{busca} / \mathrm{dataset} / \mathrm{conjunto-de-datos-vectoriales-de-carreteras-y-vialidades-urba-}$ nas-edicion-1-0.

Instituto Nacional de Estadística y Geografía, 2015, Red Nacional de Caminos. 8/08/2020. Disponible en https://datos.gob.mx/busca/dataset/red-nacional-de-caminos-rnc/resource/17357ef9-ceb6-45dc-a37c-60ad728557d4

Instituto Nacional de Estadística y Geografía, 2013, Censo de Población y Vivienda. 13/08/2020. Disponible en https://www.inegi.org.mx/programas/ccpv/2010/

Jost, L., 2006, "Entropy and diversity", en OIKOS. 113(2). Fecha de consulta: 19/08/2020. pp. 363-375. Disponible en https://doi.org/10.1111/j.2006.00301299.14714.x

Lampin-Maillet, C. et al., 2010, "Mapping wildland-urban interfaces at large scales integrating housing density and vegetation aggregation for fire prevention in the South of France", Journal of Environmental Management. Fecha de publicación: enero/febrero-2010. 91(3). Fecha de consulta: Agosto-2020. pp. 732-741. Disponible: 10.1016/j.jenvman.2009.10.001.

Lezama, J. L., 1998, Teoría social, espacio y ciudad. El Colegio de México: Ciudad de México.

Méndez-Lemus, Y. y Vieyra, A., 2014, Periurbanización, exclusión y pobreza: un análisis de la población oriunda tradicionalmente dedicada a las actividades agropecuarias en los espacios periurbanos, en Guillermo, Adrián y Escamilla, I. (ed.) Segregación Urbana y Espacio de Exclusión. Ejemplos de México y América Latina. UNAM-Porrúa: Ciudad de México, pp. 379-401.

OECD, 2009, Regional typology: Updated statistics. Consulta 19/08/2020. Disponible en http://dx.doi.org/10.1787/region-data-en. 
Piorr, A., Ravetz, J. y Tosics, I., 2011, Peri-urbanisation in Europe: Towards a European Policy to Sustain Urban-Rural Futures, Plurel: Dinamarca. Disponible en 10.1021/jp070938f

Pryor, R., 1968, "Defining the Rural-Urban Fringe", en Social Forces. 47(2). Fecha de consulta: 19/08/2020. pp. 202-215. Disponible en https://doi.org/10.1093/ $\mathrm{sf} / 47.2 .202$

Ramachandra, T. V, Bharath, A. H. y Sowmyashree, M. V., 2015, "Monitoring urbanization and its implications in a mega city from space: Spatiotemporal patterns and its indicators", en Journal of Environmental Management. 148. Fecha de consulta: 19/08/2020. pp. 67-81. Disponible en https://doi.rg/10.1016/j.jenvman.2014.02.015

Ravetz, J., Fertner, C. y Nielsen, T., 2013, "Peri-urban futures: scenarios and models for land use chage in Europe", in Nilsson, K.; Pauleit, S.; Bell, S.; Aalbers, C. and Nielsen, T. (ed.), The dynamics of peri-urbanitation. Springer, pp. 13-44.

Reginster, I. y Rounsevell, M., 2006, "Future scenarios of urban land use in Europe", in Environment and planning B: Planning and Design. 33(4). Fecha de consulta: 19/08/2020. pp. 619-636. Disponible en https://doi.org/10.1068/b31079

Secretaría de Desarrollo Social; Consejo Nacional de Población, 2012, Sistema Urbano Nacional 2012. Gobierno Federal: Distrito Federal, México.

Secretaría de Desarrollo Social, 2011, La expansión de las ciudades 1980-2010. Secretaría de Desarrollo Social: Distrito Federal.

Vieyra, A., Méndez-Lemus, Y. y Hernández-Guerrero, J., 2016, Colecciones Centro de Investigaciones en Geografía Ambiental, en Vieyra, A., Méndez-Lemus, Yadira and Hernández-Guerrero, J. (eds.) Procesos urbanos, pobreza y ambiente, implicaciones en ciudades medias y megaciudades. Universidad Nacional Autónoma de México: Ciudad de México.

Xu F., 2004, Modelling the spatial pattern of urban fringe. International Institute for Geo-Information Science and Earth Observation Enschede, The Netherlands: Wuhan. Disponible en https://webapps.itc.utwente.nl/librarywww/papers_2004/ msc/upla/xu_feng.pdf

\section{RESUMEN CURRICULAR DE LOS AUTORES}

\section{Salomón González Arellano}

Doctor en Ordenamiento Territorial y Desarrollo Regional, por la Universidad Laval, en Quebec, Canadá. Profesor investigador del Departamento de Ciencias Sociales de la Universidad Autónoma Metropolitana, Unidad Cuajimalpa en la Ciudad de México. Es miembro fundador del Laboratorio de Análisis Socioterritorial y miembro del Sistema Nacional de Investigadores desde 2006. Sus investigaciones abordan temas como la morfología urbana, segregación, la accesibilidad y la movilidad. Es coordinador de la 
Red de Estudios de Forma Urbana (REFU) e investigador del Consorcio Internacional de Hungry Cities. Salomón es cofundador de la Red Internacional de Investigación "Villes du Futur" financiada por CNRS-Francia (2020-2029) y coordinador del proyecto de investigación "Ciudades en Transición" financiado por CONACYT 2020-2023. Entre sus publicaciones más importantes destacan: La forma urbana actual de las zonas metropolitanas en México: indicadores y dimensiones morfológicas (en coautoría con Larralde, A.) y Conceputalización y medición de lo rural. Una propuesta para clasificar el espacio rural en México (en coautoría con Larralde, A.).

Dirección electrónica: sgonzalez@cua.uam.mx

Registro ORCID: https://orcid.org/0000-0002-4890-2199

\section{Adriana Helia Larralde Corona}

Maestra en Desarrollo Urbano por El Colegio de México y Doctora en Ciencias Sociales con especialidad en Sociología por la misma institución. Ha trabajado en diversas dependencias del gobierno federal y estatal. Desde el año 2017 es profesora-investigadora de tiempo completo de la Universidad Autónoma Metropolitana, unidad Cuajimalpa. Entre sus publicaciones destacan La forma urbana actual de las zonas metropolitanas en México: indicadores y dimensiones morfológicas (en coautoría con González, S.) y La configuración socio-espacial del trabajo rural y las relaciones campo-ciudad: dos localidades del centro de México.

Dirección electrónica: alarralde@cua.uam.mx

Registro ORCID: https://orcid.org/0000-0003-0807-1615

Gustavo Manuel Cruz Bello

Gustavo Manuel Cruz Bello es doctor en Estudios de los Recursos Naturales Renovables por la Universidad de Arizona, EUA, maestro en ecología y licenciado en biología por la Universidad Nacional Autónoma de México. Ha sido Director de Integración Regional en la SEMARNAT, 2002 a 2003; Secretario Ejecutivo del Programa de Cooperación en Investigación y Tecnología para la Región Norte del IICA, 2004 a 2009. Actualmente es Profesor-Investigador en el Departamento de Ciencias Sociales de la Universidad Autónoma Metropolitana. Es miembro del Sistema Nacional de Investigadores. Entre sus publicaciones más importantes destacan: Flood vulnerability reduction, using a partial participatory GIS approach. A study case in Baja California Sur, Mexico; Coupling spatial multiattribute analysis and optimization to identify reforestation priority areas: A case 
study in central Mexico y Linking multi-temporal analysis and community consultation to evaluate the response to the impact of Hurricane Stan in coffee areas of Chiapas, Mexico.

Dirección electrónica: gcruz@cua.uam.mx

Registro ORCID: https://orcid.org/0000-0001-9118-6565

Artículo recibido el 19 de octubre de 2020 y aprobado el 9 de julio de 2021. 
El periurbano en México: Identificación y caracterización ... / S. GONZÁLEZ ARELLANO et al.

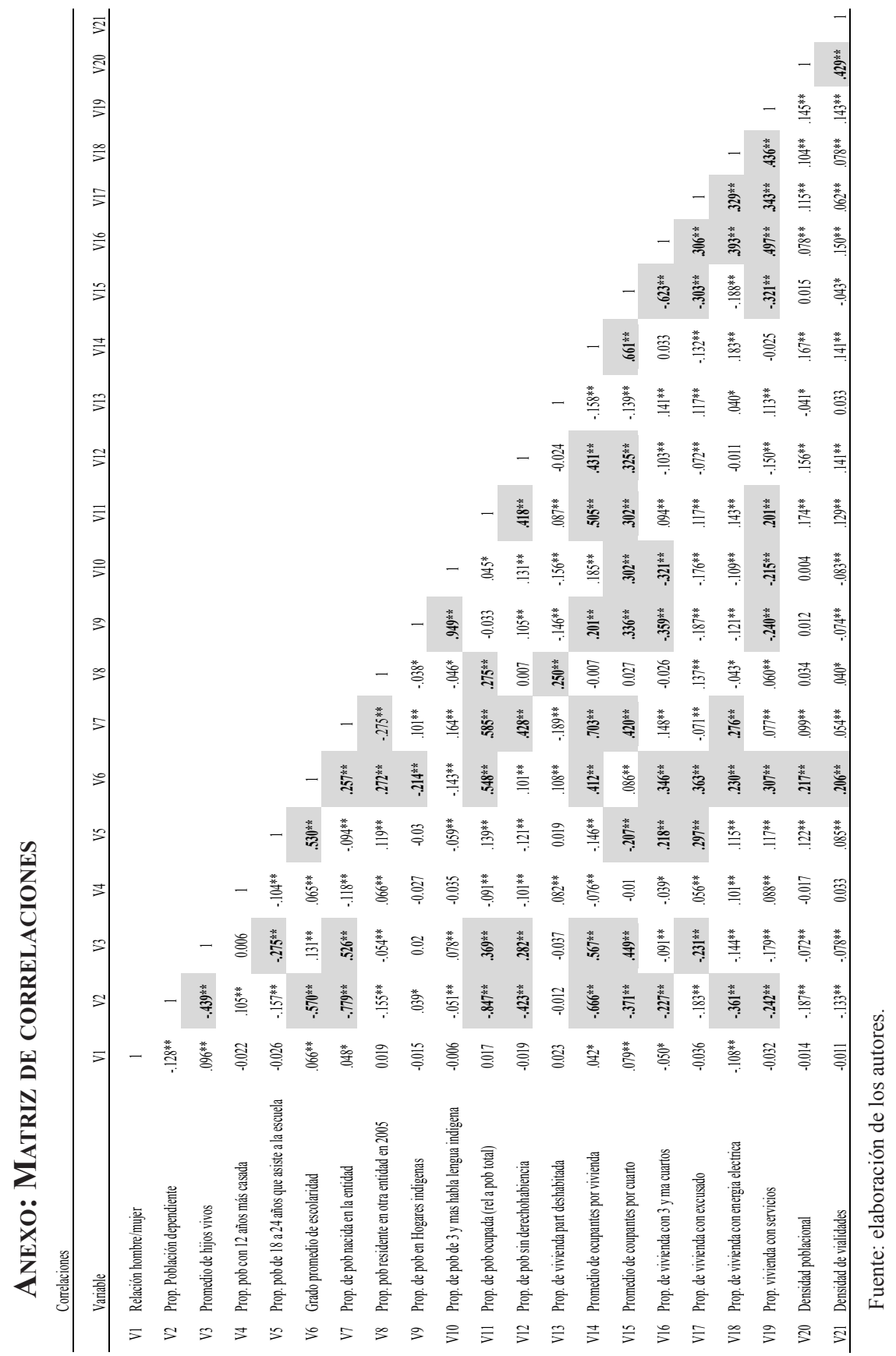

